\title{
Os processos de inovação aberta: uma análise na indústria brasileira de semicondutores
}

\begin{abstract}
Nos últimos anos, para conseguir inovar, as empresas tem se tornado cada vez mais dependentes de conhecimentos e recursos gerados fora das fronteiras organizacionais. Assim, o processo de inovação nas organizações passou a considerar a contribuição de diferentes agentes externos, dentre eles clientes, fornecedores e universidades. Na perspectiva da inovação aberta, segundo Gassman et al. (2004), os processos de inovação podem ser categorizados em três tipos: de entrada ou outside-in, de saída ou inside-out, e por fim, o processo acoplado ou coupled process, sendo esses arquétipos caracterizados pela adoção de diferentes atividades. O presente artigo teve como objetivo identificar os processos de inovação aberta adotados pelas design houses brasileiras no desenvolvimento conjunto de projetos de circuitos integrados, para isso, foi utilizado o survey como método de pesquisa, com a realização de análise documental e a aplicação de questionários estruturados com design houses e especialistas da área de microeletrônica. Os dados foram analisados com o auxílio do programa SPSS (versão 21), por meio da utilização de técnicas descritivas e estatísticas, dentre elas a aplicação do teste do qui-quadrado. Os resultados mostraram que as design houses estudadas implementam diversas práticas do paradigma de inovação aberta no desenvolvimento de projetos de circuitos integrados, sendo as práticas referentes ao processo de entrada as mais frequentes, além disso, foi possível observar que não existiam diferenças significativas quando a design house possui ou não fins lucrativos e os processos de inovação aberta adotados.
\end{abstract}

Palavras-chave: Inovação Aberta; Processos; Semicondutores; Design Houses.

\section{The processes of open innovation: an analysis in the brazilian semiconductor industry}

Over the past years, in order to innovate, companies have become increasingly dependent on knowledge and resources generated outside organizationa boundaries. Thus, the process of innovation in organizations began to consider the contribution of different external agents, among them clients, suppliers, and universities. In the perspective of open innovation, according to Gassman et al. (2004), innovation processes can be categorized into three types: outside-in, insideout and coupled process, being these archetypes characterized by the adoption of different activities. The present article aimed to identify the processes of open innovation adopted by Brazilian design houses in the joint development of integrated circuit projects, for that, the research method applied was a survey, using documentary analysis and the application of structured questionnaires with design houses and specialists in microelectronics. The program SPSS (version 21) was used to help the data analysis, using descriptive and statistical techniques, among them the application of the chi-square test. The study results showed that the design houses studied implement several practices of the open innovation paradigm in the development of integrated circuits projects, being the practices related to the outside-in process the most frequent, in addition, it was possible to observe there were no significant differences when the design house was a for-profit or nonprofit organization and the open innovation processes adopted.

Keywords: Open Innovation; Processes; Semiconductors; Design Houses.

Topic: Inovação Tecnológica

Reviewed anonymously in the process of blind peer
Received: 21/10/2017

Approved: 24/12/2017
Xênia L'amour Campos Oliveira (it) Universidade Federal de Sergipe, Brasil http://lattes.cnpq.br/8152406830012615 http://orcid.org/0000-0003-4178-6006 xenia.Ico@gmail.com

\section{Maria Elena Léon Olave}

Universidade Federal de Sergipe, Brasil http://lattes.cnpq.br/9409501680710062 mleonolave@gmail.com
Referencing this:

OLIVEIRA, X. L. C.; OLAVE, M. E. L.. Os processos de inovação aberta: uma análise na indústria brasileira de semicondutores. Revista Brasileira de Administração Científica, v.8, n.3, p.158-170, 2017. DOI: http://doi.org/10.6008/SPC2179-684X.2017.003.0013 


\section{INTRODUÇÃO}

As empresas investem uma quantidade significativa de tempo, dinheiro e outros recursos, com a finalidade de buscar novas oportunidades inovadoras, e esse investimento amplia sua capacidade de criar, utilizar e recombinar conhecimentos existentes e novos (LAURSEN et al., 2006). As constantes mudanças por meio das inovações tecnológicas, tornaram pouco provável que uma empresa consiga sustentar sua vantagem frente às outras sem fazer uso de tecnologias e conhecimentos externos (ASAKAWA et al., 2014). Fatores como o aumento da mobilidade humana, o dinamismo do mercado e as rápidas mudanças nas tecnologias de informação, fizeram com que os conhecimentos que contribuem para o processo de inovação de uma empresa estejam disponíveis e dispersos em diferentes países, organizações e pessoas (SEGARRACIPRÉS et al., 2012).

O fluxo de conhecimentos mobilizado entre as organizações tornou-se essencial para o processo de inovação (SIMARD et al., 2011), razão pela qual nos últimos anos, em busca de vantagens competitivas, as empresas tem utilizado cada vez mais fontes internas e externas de recursos, buscando tecnologias de outras empresas, e gerando receitas por meio de contratos de licenciamento ou spin-offs de tecnologias não exploradas internamente (SCHNECKENBERG, 2015; VANHAVERBEKE et al., 2014; ZAHRA et al., 2002).

Diante desse contexto, Chesbrough (2012) apresentou o paradigma da inovação aberta, no qual as empresas podem utilizar tanto recursos internos quanto externos para desenvolver uma inovação. Para Dahlander et al. (2010), a abertura do processo de inovação é fundamentada na ideia de que uma única organização não pode inovar isoladamente, sendo assim, Chesbrough et al. (2014) reconhece que, no modelo de inovação aberta, os fluxos de conhecimento podem admitir essencialmente dois sentidos, são eles: o de entrada ou outside-in e o de saída ou inside-out, aos quais Gassmann et al. (2004) acrescentou um terceiro tipo, que interliga esses dois processos, denominado processo de inovação acoplado ou coupled process.

Cada um desses processos ou arquétipos representam uma estratégia na perspectiva da inovação aberta, e estão relacionados a diferentes práticas que podem ser implementadas pelas empresas, dentre elas a integração de conhecimento externo e o desenvolvimento de inovações por meio de alianças estratégicas (GASSMAN et al., 2004).

Nas indústrias intensivas em conhecimento, como a indústria eletroeletrônica, e especificamente, a de semicondutores (WU et al., 2015), fatores como a rapidez das inovações no setor tem apontado que a maioria das inovações são desenvolvidas a partir de projetos colaborativos (BAHINIPATI et al., 2012; BROWN et al., 2009; KAPOOR et al., 2014).

Apesar das contribuições do modelo de inovação aberta (IA) para a indústria de semicondutores (CHESBROUGH, 2012; MÜLLER-SEITZ et al., 2012), os estudos nessa indústria tem se concentrado em temas como: cooperação universidade-empresa (OLIVEIRA et al., 2015), projetos colaborativos (FACCIN et al., 2015; FACCIN et al., 2016; KAPOOR et al., 2014; SONG, 2011), redes de inovação (LANGE et al., 2013; PERRI et al., 2014), e políticas públicas de fomento à indústria (FACCIN et al., 2016; HAUSER et al., 2007; WANG et al., 2014). Diante disso, convém que mais estudos que abordem o fenômeno da inovação aberta na indústria de semicondutores, especialmente no âmbito brasileiro, sejam realizados. 
No contexto brasileiro, a indústria de semicondutores é considerada de grande potencial quanto à criação de vantagens competitivas, sendo vista como um motor de inovação e desenvolvimento tecnológico (ABDI, 2011; BRASIL, 2002; LIMA et al., 2015). Dentro do contexto da cadeia produtiva de semicondutores, o foco de análise deste estudo foi nas design houses, empresas responsáveis pela definição das funcionalidades dos chips que serão incorporados aos produtos finais, sendo consideradas agentes impulsionadores da inovação na indústria (AITA, 2013; FACCIN et al., 2015).

Em função do contexto apresentado sobre a indústria de semicondutores e as possibilidades dispostas pelo modelo de inovação aberta, e como forma de contribuir para uma maior compreensão acerca da adoção das práticas de inovação aberta na indústria de semicondutores, este artigo buscou identificar os processos de inovação aberta adotados pelas design houses brasileiras no desenvolvimento conjunto de projetos de circuitos integrados.

\section{REVISÃO TEÓRICA}

\section{Tipos de inovação aberta}

Com o objetivo de situar o campo de pesquisa sobre inovação aberta, a fundamentação teórica a seguir apresenta os tipos de processos de inovação aberta que podem ser adotadas pelas empresas. De acordo com Chesbrough et al. (2014), na perspectiva do modelo de inovação aberta, os fluxos de conhecimento podem admitir essencialmente dois sentidos, são eles: o de entrada ou outside-in, e o de saída ou inside-out. Estes, constituem tipos de inovação aberta, aos quais Gassmann et al. (2004) acrescentou um terceiro tipo, o processo de inovação acoplado ou coupled process.

Segundo Gassmann et al. (2004), no processo de entrada/outside-in, o principal objetivo é enriquecer a base de conhecimentos da organização, aumentando a capacidade da empresa em inovar, através da integração do conhecimento externo de fornecedores, clientes, e outros agentes externos. Já no processo de saída/inside-out, as empresas encaminham as ideias para o mercado e comercializam propriedade intelectual, com a intenção de obter lucros.

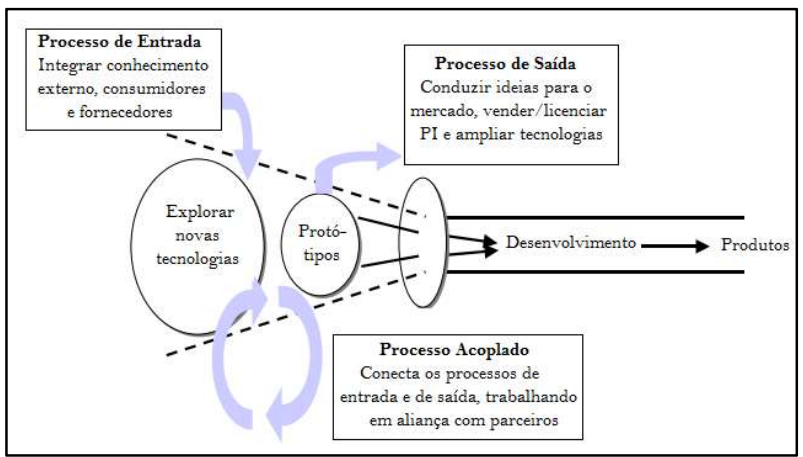

Figura 1: Tipos de processos de inovação aberta. Fonte: Gassmann et al. (2004).

No processo de inovação acoplado/coupled, as organizações optam por empregar ambas as estratégias de entrada e de saída, através de alianças com parceiros, nas quais, a troca de informações é essencial para o sucesso da parceria. Para os autores, a ênfase dada a esses processos pode variar de acordo com a organização, nem todas as empresas adotam os mesmos processos de inovação aberta, ou integram 
os três processos simultaneamente. Normalmente a empresa prioriza um dos processos, e integra alguns elementos dos demais. A figura 1 exibe os três tipos de inovação aberta, explanando, resumidamente, suas principais finalidades.

No processo de entrada ou outside-in, a empresa opta por investir em cooperação (GASSMANN et al., 2004), ela "abre" seu processo de inovação a diferentes tipos de entradas e contribuições externas (CHESBROUGH et al., 2014). As fontes de conhecimentos externas podem ser empregadas para auxiliar o desenvolvimento tecnológico atual da empresa, e o envolvimento dos clientes, empresas parceiras, além de universidades e centros de pesquisa, podem ajudar a acelerar o processo de inovação da empresa (VRANDE et al., 2009).

Dentre os mecanismos através dos quais as empresas podem gerenciar os fluxos de conhecimento externo, estão: a colaboração com fornecedores e clientes, os programas de pesquisa de universidades, o financiamento de startups em uma determinada indústria; ou até mesmo mecanismos mais recentes, como o crowdsourcing ${ }^{1}$ ou os spin-ins ${ }^{2}$ (CHESBROUGH, 2011; CHESBROUGH et al., 2014).

Entre as principais vantagens em utilizar fontes externas de inovação estão o acesso a recursos únicos, e a conhecimentos novos e complementares. Os clientes e fornecedores representam fontes de conhecimento e competência relevantes para o processo de desenvolvimento de produtos, além de patentes e conhecimento tecnológico obtido através de polos regionais de inovação, estes polos, ou centros de excelência, são resultado de investimentos estrangeiros realizados pelas empresas para acessar conhecimentos e explorar recursos (GASSMANN et al., 2004).

Para Nigro (2016), devido às incertezas que envolvem a atividade de P\&D, o processo de entrada é um exercício desafiador. Dentre os tipos de empresas que mais se beneficiam em adotar a estratégia de entrada ou outside-in como base para seu processo de inovação, estão: as indústrias de baixa tecnologia, como por exemplo as empresas de ar-condicionado e processos de automação, que aguardam os spillovers da indústria de alta tecnologia; indústrias de produtos altamente modulados, como por exemplo, empresas de elevadores; e, indústrias de alta intensidade tecnológica, já que suas necessidades não podem ser atendidas empregando apenas suas capacidades e conhecimentos internos, exemplos de empresas incluem, a 3M, a Procter \& Gamble e a Siemens (GASSMANN et al., 2004).

As empresas que adotam como estratégia de inovação o processo de saída ou inside-out, possuem como propósito de levar ideias para o mercado mais rapidamente do que elas conseguiriam utilizando apenas seus recursos internos (ENKEL et al., 2009), as capacidades tecnológicas da empresa são exploradas fora das fronteiras organizacionais, por meio da criação de novos empreendimentos e do licenciamento de propriedade intelectual para o ambiente externo (VRANDE et al., 2009), sustentando a ideia de que o lócus da inovação ou da invenção, não será, necessariamente, o mesmo lócus de sua exploração (GASSMANN; ENKEL, 2004).

\footnotetext{
${ }^{1} \mathrm{O}$ crowdsourcing é uma prática de negócios na qual as empresas, ao invés de tentar resolver problemas internamente, poderão fazer uso de plataformas online, onde qualquer pessoa poderá submeter suas ideias e soluções criativas para o problema da empresa (PÉNIN et al., 2011).

${ }^{2}$ Os spin-ins são mecanismos de aquisição de tecnologias externas, eles podem ser verificados quando uma organização adquire participações minoritárias, realiza acordos de licenciamento, ou outros tipos de mecanismos de relação para acessar tecnologias de outra empresa (TIDD et al., 2000).
} 
Neste tipo de inovação aberta, a empresa não fica mais restrita ao mercado que atua diretamente (ENKEL et al., 2009), portanto, ela deve permitir que ideias e ativos não utilizados ou subutilizados por ela, sejam explorados por outras empresas, por meio de mecanismos como: licenciamento e doação de tecnologia, spin-offs, joint ventures, e incubadoras de empresas (CHESBROUGH et al., 2014).

A comercialização de ideias em diversas indústrias pode favorecer consideravelmente o aumento das receitas de uma empresa. Normalmente, empresas que adotam o processo de saída como suporte para seu processo de inovação são orientadas para pesquisa básica, com aplicações diversas, como por exemplo a IBM, e cujo objetivos são reduzir os custos fixos em P\&D e compartilhar riscos. Além destas, as empresas que empregam a estratégia de branding (verificada quando uma empresa utiliza a marca e as competências de outras organizações), para o desenvolvimento e comercialização de uma tecnologia, também podem justificar a adoção do processo de inside-out (GASSMANN et al., 2004).

A terceirização de uma tecnologia ou a criação de parcerias de apoio para o desenvolvimento de uma nova tecnologia ou conhecimento, pode ser resultado da intenção de uma empresa em determinar padrões tecnológicos. Os spillovers são efeitos positivos de uma inovação, que podem ser comercializados em outras indústrias, por exemplo, processadores que para a indústria de TI são tecnologias ultrapassadas, podem ser integrados com sucesso em outros produtos como elevadores ou carros (CHESBROUGH, 2011; GASSMANN et al., 2004).

No processo de inovação aberta acoplado ou coupled process, os processos de saída e de entrada, discutidos anteriormente, são interligados, assim, a combinação de fluxos de entradas e saídas de conhecimentos são empregados para desenvolver e/ou comercializar uma inovação de forma colaborativa (CHESBROUGH et al., 2014), para que isto ocorra, as empresas cooperam com outras organizações por meio de redes estratégicas (GASSMANN et al., 2004).

O conceito de cooperação faz referência ao desenvolvimento de conhecimentos de forma conjunta. As empresas podem desenvolver relacionamentos de cooperação com fornecedores, clientes, universidades e centros de pesquisa, além de seus próprios competidores (GASSMANN et al., 2004; SMIRNOVA et al., 2009). Essa cooperação, em princípio, pode ocorrer através da combinação de mecanismos aplicáveis aos processos de inovação aberta de saída ou de entrada, contudo, as empresas podem aplicar mecanismos específicos, como por exemplo, alianças estratégicas, consórcios, joint ventures, redes, ecossistemas e plataformas (CHESBROUGH et al., 2014).

A maioria das empresas que adotam o processo de inovação aberta acoplado tem por objetivo definir normas ou projetos dominantes para seus produtos, por exemplo, empresas podem formar alianças para definir e desenvolver um padrão para determinada tecnologia, como pode ser observado na produção de chips de identificação por radiofrequência (RFID), por meio da aliança de empresas como a Unilever e a Henkel (GASSMANN et al., 2004). Gassmann et al. (2004) cita ainda que empresas que buscam alcançar retornos crescentes pela exploração múltipla de uma inovação, devem escolher como suporte para inovar, o coupled process, por exemplo, a indústria de telefonia móvel, onde novas tecnologias como o MMS ou os 
ring tunes são introduzidas, devem trabalhar em aliança com as empresas de telecomunicações para garantir que suas tecnologias sejam implementadas em novas gerações de telefones móveis.

A figura 2 apresenta os processos de inovação aberta e as respectivas atividades que auxiliam a caracterizar a estratégia adotada pela empresa. Ainda referente aos tipos de inovação aberta, estudos como o de Lichtenthaler et al. (2009), Vrande et al. (2009), Dahlander et al. (2010), e Huizingh (2011) apresentam outras perspectivas quanto à sua classificação.

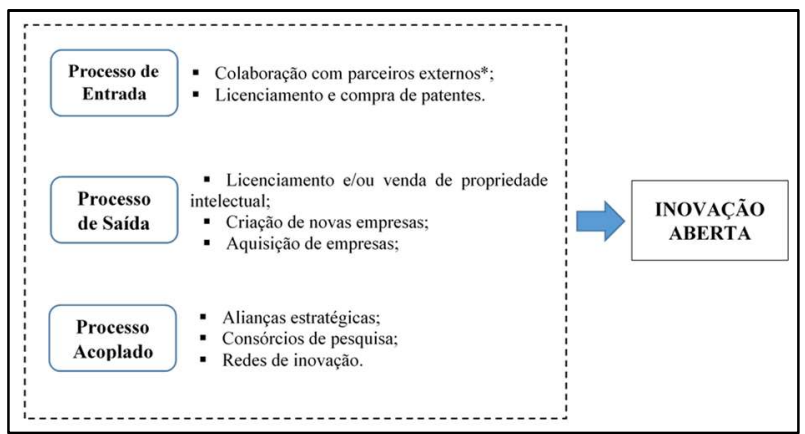

Figura 2: Processos de inovação aberta e suas atividades. Fonte: Gassmann et al. (2004).

Nota: Os parceiros externos podem ser: clientes, concorrentes, institutos de pesquisa, consultores, fornecedores, governo, universidades ou outras empresas públicas.

\section{METODOLOGIA}

A fim de investigar quais os processos de inovação aberta adotados pelas design houses brasileiras, o método de pesquisa aplicado neste estudo foi o survey. As pesquisas do tipo survey fornecem técnicas que permitem estudar "quase todo mundo", esse mundo é representado pelas unidades de análise (BABBIE, 2003, p. 98), que na presente pesquisa, são representadas pelas design houses participantes do Programa ClBrasil e especialistas da área de microeletrônica (professores, engenheiros e pessoas que possuem experiência na indústria, seja profissional ou academicamente).

Esta pesquisa adotou a survey do tipo interseccional, os dados foram coletados de uma população em particular, em um determinado momento no tempo, com o intuito de ajudar a descrever e determinar as relações existentes entre as variáveis na época do estudo (BABBIE, 2003).

Os instrumentos de coleta de dados adotados foram a análise documental e a aplicação de questionários estruturados. Em relação ao tipo de questionário adotado, optou-se pelo tipo auto administrado mediado pela internet, com questões fechadas e escala nominal (SAUNDERS et al., 2009). 0 questionário foi elaborado em duas versões com o auxílio da plataforma online 'Formulários' do Google uma das versões foi enviada por e-mail às empresas e a outra aos especialistas da área.

Para obter uma maior validade das questões e confiabilidade dos dados que foram coletados, foi realizado um pré-teste do questionário, este foi submetido a análise de um especialista da área, foram sugeridas algumas mudanças e o tipo de escala adotada foi modificada, de uma escala ordinal para nominal (HILL et al., 2012). Depois de realizadas as alterações, o questionário foi enviado a duas empresas e a dois especialistas para teste, duas respostas foram obtidas (uma empresa e um especialista), os respondentes não informaram dificuldades e não sugeriram mudanças quanto ao instrumento de pesquisa. 
No que diz respeito à análise documental, esta compreendeu o exame das informações contidas nos sites das design houses, como por exemplo, contatos ou produtos oferecidos, além de documentos do governo sobre a indústria, como panoramas econômicos e relatórios, estes foram retirados dos sites da Agência Brasileira de Desenvolvimento Industrial (ABDI), da Associação Brasileira da Indústria Elétrica e Eletrônica (ABINEE), além de outros órgãos governamentais, como por exemplo o Ministério da Ciência, Tecnologia e Inovação (MCTI).

Com o intuito de enriquecer a coleta dos dados desta pesquisa e possibilitar a comparação dos resultados obtidos a partir das respostas coletadas sobre a realidade das empresas estudadas, a população foi composta pelas 22 (vinte e duas) design houses participantes do Programa Cl-Brasil e especialistas da área de microeletrônica.

No entanto, no momento da pesquisa, apenas dezenove das design houses vinculadas ao programa possuíam site e informações de contato disponíveis na internet, ainda de acordo com a ABDI (2014), dessas empresas somente dezoito continuavam operando normalmente, as empresas DHBH, Floripa DH, Minas IC e TE@I2 haviam encerrado ou paralisado suas atividades. Dessa forma, foram enviados questionários a todas as 19 (dezenove) design houses e a alguns especialistas, sendo os últimos selecionados por conveniência (HILL et al., 2012). Assim, a amostra do estudo foi composta por 11 (onze) DHs e 21 (vinte e um) especialistas da área.

Após a coleta dos dados, foi utilizada a ferramenta 'ver as respostas no Planilhas' da plataforma 'Formulários', que insere automaticamente os dados coletados no programa online 'Planilhas' do Google ${ }^{\circledR}$, a partir do qual foi possível realizar o download das respostas para o Excel, permitindo assim que os dados fossem transferidos para o programa SPSS (Statistical Package for Social Sciences) versão 21 para análise mais completa. Para análise dos dados foram utilizadas técnicas descritivas e estatísticas, foi efetuado o teste de normalidade, e como os dados da pesquisa não seguiam a hipótese da normalidade, para averiguar a associação ou independência entre as variáveis foi aplicado o teste do qui-quadrado.

\section{RESULTADOS E DISCUSSÃO}

Nessa primeira parte da análise são apresentados os resultados referentes à caracterização das design houses (DHs) associadas ao programa $\mathrm{Cl}$-Brasil participantes da pesquisa e os resultados de algumas perguntas específicas feitas a essas DHs. Como foi relatado na metodologia, dos questionários respondidos, um (01) precisou ser descartado por não atender aos requisitos da pesquisa, já que muitas questões foram deixadas sem resposta. Com o intuito de facilitar o controle das respostas e um possível contato posterior, foi solicitada a identificação das empresas respondentes, e com essa informação foi possível detectar a natureza jurídica das DHs. A tabela 01 apresenta informações sobre a natureza jurídica e o número de funcionários das DHs.

Quanto a natureza jurídica, foi possível constatar que cinco empresas possuíam fins lucrativos, enquanto as outras seis são instituições sem fins lucrativos. Com relação ao número de funcionários, mais da metade das empresas respondentes $(54,5 \%)$ contam com um quadro de até 20 colaboradores, informações 
similares foram apontadas no relatório realizado pela ABDI (2014), segundo o qual, no geral as DHs brasileiras são consideradas de pequeno porte, quando se refere ao número de funcionários. De acordo com informações das empresas, no que diz respeito ao nível de formação, em sua maioria esses colaboradores são graduados nas áreas de engenharia, e alguns possuem mestrado e doutorado em diversas áreas.

Tabela 1: Perfil institucional das design houses.

\begin{tabular}{|c|c|c|}
\hline Design house & Natureza jurídica & Número de funcionários da empresa \\
\hline DH 1 & Com fins lucrativos & Entre 21 e 50 \\
\hline DH 2 & Com fins lucrativos & Enos de 5 \\
\hline DH 3 & Com fins lucrativos & Mais de 100 \\
\hline DH 4 & Com fins lucrativos & Entre 11 e 20 \\
\hline DH 5 & Com fins lucrativos & Mais de 100 \\
\hline DH 6 & Sem fins lucrativos & Entre 05 e 10 \\
\hline DH 7 & Sem fins lucrativos 51 e 100 \\
\hline DH 8 & Sem fins lucrativos & Entre 11 e 20 \\
\hline DH 9 & Sem fins lucrativos & Entre 11 e 20 \\
\hline DH 10 & Sem fins lucrativos & Entre 11 e 20 \\
\hline DH 11 & Sem fins lucrativos & \\
\hline
\end{tabular}

O questionário aplicado descreveu a adoção de algumas situações que ajudariam a determinar qual o processo de inovação aberta adotada pelas DHs (ver figura 2): sete (07) questões abordaram situações referentes ao processo de entrada; seis (06) questões salientaram situações relacionadas ao processo de saída; e por fim, 05 (cinco) questões abordaram situações relativas ao processo acoplado. Para facilitar a análise dos dados, foram atribuídos os rótulos de 'Especialista: Esp_ $n$ ' e 'Empresa: DH_ $n$ ' para os respondentes, e as respostas afirmativas dadas às questões que abordaram as diferentes características dos três processos de inovação aberta foram contabilizadas. A tabela 2 apresenta os resultados das respostas das design houses.

Tabela 2: Frequências observadas: empresa versus inovação.

\begin{tabular}{|c|c|c|c|c|}
\hline \multicolumn{5}{|c|}{ Empresa * Inovação - Tabulação cruzada } \\
\cline { 2 - 5 } Empresa & Acoplado & Entrada & \multirow{2}{*}{ Total } \\
\cline { 2 - 5 } & 1 & 7 & 2 & 10 \\
\hline DH_01 & 4 & 6 & 4 & 13 \\
\hline DH_02 & 2 & 7 & 2 & 13 \\
\hline DH_03 & 0 & 3 & 1 & 5 \\
\hline DH_04 & 3 & 7 & 5 & 11 \\
\hline DH_05 & 3 & 7 & 1 & 15 \\
\hline DH_06 & 1 & 3 & 4 & 5 \\
\hline DH_07 & 5 & 4 & 2 & 13 \\
\hline DH_08 & 5 & 5 & 3 & 12 \\
\hline DH_09 & 3 & 5 & 2 & 11 \\
\hline DH_10 & 2 & 7 & 29 & 11 \\
\hline DH_11 & 29 & 61 & & 119 \\
\hline Total & & & & 2 \\
\hline
\end{tabular}

Já a tabela 3 divide as DHs quanto a sua natureza jurídica, pode-se observar que o processo acoplado é o que apresenta a maior diferença entre as empresas com fins lucrativos (34,5\%) e sem fins lucrativos $(65,5 \%$ ), seguido pelo processo de saída (com fins lucrativos - 41,4\%; sem fins lucrativos $-58,6 \%$ ), e o processo de entrada, no qual os percentuais apresentados foram bastante similares (com fins lucrativos 49,2\%; sem fins lucrativos $-50,8 \%$ ). A tabela 4 apresenta os resultados das respostas dos especialistas, podese observar que apenas um dos participantes, o 'Esp_8', respondeu negativamente a todas as questões. 
Tabela 3: Frequências observadas: natureza jurídica versus inovação.

\begin{tabular}{|c|c|c|c|}
\hline \multicolumn{3}{|c|}{ Natureza jurídica * Inovação - Tabulação cruzada } \\
\hline \multirow{2}{*}{ Natureza Jurídica } & \multicolumn{3}{|c|}{ Processo de Inovação Aberta } \\
\cline { 2 - 4 } & Acoplado & Entrada & Saída \\
\hline Com fins lucrativos & $34,5 \%$ & $49,2 \%$ & $41,4 \%$ \\
\hline Sem fins lucrativos & $65,5 \%$ & $50,8 \%$ & $58,6 \%$ \\
\hline Total & $100 \%$ & $100 \%$ & $100 \%$ \\
\hline
\end{tabular}

Tabela 4: Frequências observadas: especialista versus inovação.

\begin{tabular}{|c|c|c|c|c|}
\hline \multicolumn{5}{|c|}{ Especialista * Inovação - Tabulação cruzada } \\
\hline \multirow{2}{*}{ Especialista } & \multicolumn{3}{|c|}{ Processo de Inovação Aberta } & \multirow{2}{*}{ Tota } \\
\hline & Acoplado & Entrada & Saída & \\
\hline Esp_1 & 1 & 3 & 1 & 5 \\
\hline Esp_2 & 4 & 7 & 5 & 16 \\
\hline Esp_3 & 4 & 5 & 2 & 11 \\
\hline Esp_4 & 5 & 7 & 3 & 15 \\
\hline Esp_5 & 2 & 6 & 0 & 8 \\
\hline Esp_6 & 1 & 5 & 2 & 8 \\
\hline Esp_7 & 1 & 6 & 1 & 8 \\
\hline Esp_8 & 0 & 0 & 0 & 0 \\
\hline Esp_9 & 2 & 5 & 0 & 7 \\
\hline Esp_10 & 2 & 3 & 1 & 6 \\
\hline Esp_11 & 4 & 7 & 4 & 15 \\
\hline Esp_12 & 1 & 7 & 3 & 11 \\
\hline Esp_13 & 4 & 3 & 4 & 11 \\
\hline Esp_14 & 1 & 4 & 3 & 8 \\
\hline Esp_15 & 3 & 5 & 1 & 9 \\
\hline Esp_16 & 4 & 5 & 1 & 10 \\
\hline Esp_17 & 0 & 2 & 2 & 4 \\
\hline Esp_18 & 2 & 4 & 5 & 11 \\
\hline Esp_19 & 2 & 2 & 1 & 5 \\
\hline Esp_20 & 1 & 4 & 0 & 5 \\
\hline Esp_21 & 3 & 3 & 0 & 6 \\
\hline Total & 47 & 93 & 39 & 179 \\
\hline
\end{tabular}

A tabela 5 exibe as frequências totais das respostas, isto é, tanto das design houses quanto dos especialistas, para cada processo de inovação aberta, pode-se observar que a maior incidência de respostas afirmativas estava relacionada às questões que abordaram situações referentes ao processo de entrada (51,7\%), seguido pela adoção de práticas do processo acoplado (25,5\%), e por fim, a adoção de práticas do processo de saída $(22,8 \%)$.

Tabela 5: Porcentual das respostas relacionadas aos processos de inovação aberta.

\begin{tabular}{|c|c|c|c|c|}
\hline Inovação & Frequência & Porcentual & Porcentagem válida & Porcentagem acumulativa \\
\hline Acoplado & 76 & 25,5 & 25,5 & 25,5 \\
\hline Entrada & 154 & 51,7 & 51,7 & 77,2 \\
\hline Saída & 68 & 22,8 & 22,8 & 100,0 \\
\hline Total & 298 & 100,0 & 100,0 & \\
\hline
\end{tabular}

Desse modo, os resultados mostram que, de acordo com os respondentes, a adoção das práticas de inovação aberta referentes ao processo de entrada são as mais frequentes entre as design houses estudadas, como por exemplo, a colaboração com parceiros externos, dentre eles clientes, fornecedores e universidades. No entanto, algumas práticas referentes aos processos de saída e acoplado também são empregadas pelas empresas, como por exemplo, a criação de novas empresas e a participação em consórcios de pesquisa. 
O teste de Kolmogorov-Smirnov (K-S) e Shapiro-Wilk foi utilizado para analisar se as distribuições amostrais são normais ou não. Considerando um intervalo de confiança de 95\%, para aceitar a hipótese nula, o p-valor precisa ser maior que 0,05 (FIELD, 2009). 0 teste foi aplicado aos indicadores que teoricamente influenciam na adoção das práticas de inovação aberta, e o resultado mostrou que o $p$-valor dos indicadores apresentaram valores inferiores a 0,05. Deste modo, rejeita-se a hipótese nula, ou seja, os dados não atendem ao pressuposto da normalidade, diante disso, o teste a ser aplicado para testar as hipóteses do estudo deve ser o não paramétrico, dessa forma, o teste do qui-quadrado foi utilizado para testar algumas hipóteses do presente estudo.

Para investigar a existência de associação ou independência entre as variáveis: natureza jurídica e o processo de inovação aberta adotado pelas design houses, os dados apresentados na tabela 6 foram utilizados como base para aplicação do teste do qui-quadrado. Assim, as hipóteses a testadas foram: $H_{0}$ : As variáveis natureza jurídica e processo de inovação aberta são independentes; e $H_{1}$ : As variáveis natureza jurídica e processo de inovação aberta não são independentes. A tabela 6 apresenta o resultado do teste de qui-quadrado relacionados as variáveis: natureza jurídica e processo de inovação aberta.

Tabela 06: Teste do qui-quadrado para independência ou associação: natureza jurídica * inovação.

\begin{tabular}{|c|c|c|c|}
\hline & Qui-quadrado & Df & Sig. \\
\hline Qui-quadrado de Pearson & 1,810 & 2 & $\mathbf{0 , 4 0 5}$ \\
\hline Razão de verossimilhança & 1,827 & 2 & $\mathbf{0 , 4 0 1}$ \\
\hline Número de Casos Válidos & 119 & - & - \\
\hline
\end{tabular}

Conforme se apresenta na tabela 6, pode-se verificar que o nível de significância (Sig.) para o teste foi de 0,405 , um resultado superior ao padrão de 0,05 , deste modo, não se pode rejeitar a hipótese nula, aceitando-se o fato da independência das variáveis analisadas, ou seja, pode-se afirmar que não existem diferenças significativas entre a design house possuir ou não fins lucrativos e os processos de inovação aberta adotados.

O teste do qui-quadrado também foi aplicado para determinar a independência ou associação entre as variáveis 'respondente' e 'inovação', isto é, para determinar se existiam diferenças significativas no padrão das respostas das design houses e dos especialistas da área que participaram da pesquisa, e o processo de inovação aberta adotado. A tabela 7 exibe o resultado do teste do qui-quadrado para determinar a independência ou associação entre as variáveis 'respondente' e 'inovação'.

Tabela 7: Teste do qui-quadrado para independência ou associação: respondente * inovação.

\begin{tabular}{|c|c|c|c|}
\hline & Qui-quadrado & Df & Sig. \\
\hline Qui-quadrado de Pearson & 0,315 & 2 & $\mathbf{0 , 8 5 4}$ \\
\hline Razão de verossimilhança & 0,314 & 2 & $\mathbf{0 , 8 5 5}$ \\
\hline N de Casos Válidos & 298 & - & - \\
\hline
\end{tabular}

É possível observar, a partir da tabela 7, que o nível de significância para o teste foi de 0,854, ou seja, não se pode rejeitar a hipótese nula, aceitando-se o fato da independência entre as variáveis analisadas. A partir desse resultado pode-se afirmar que não existem diferenças significativas entre os respondentes da pesquisa e os tipos de inovação aberta adotadas pelas DHs brasileiras, ou seja, o padrão das respostas tanto das empresas quanto dos especialistas, no que diz respeito aos processos de inovação aberta adotados pelas DHs, não foi significativamente diferente. 


\section{CONCLUSÕES}

Embora a literatura sobre inovação aberta saliente os benefícios da implementação de suas práticas para a indústria de semicondutores, no contexto brasileiro poucos estudos focaram nas contribuições que a implementação dessas práticas poderia trazer para as empresas desse ramo. Sendo assim, o presente artigo procurou identificar os processos de inovação aberta adotados pelas design houses brasileiras no desenvolvimento conjunto de projetos de circuitos integrados.

Os resultados da pesquisa mostraram que as design houses estudadas implementam diversas práticas do paradigma de inovação aberta no desenvolvimento de projetos de circuitos integrados. A adoção das práticas de inovação aberta referentes ao processo de entrada mostraram ser as mais frequentes entre as design houses, como por exemplo a colaboração com parceiros externos com o objetivo de aumentar a capacidade de inovação da empresa, por meio da integração do conhecimento externo de fornecedores, clientes, e outros agentes externos (GASSMAN et al., 2004). Contudo, pode-se identificar que algumas práticas referentes aos processos de saída e acoplado também são empregadas pelas empresas, como por exemplo, a criação de novas empresas e a participação em consórcios de pesquisa (CHESBROUGH et al., 2014).

Também foi possível constatar através da aplicação do teste do qui-quadrado para independência ou associação, que não existem diferenças significativas entre a design house possuir ou não fins lucrativos e os tipos de processos de inovação aberta adotados. Além de não existirem diferenças significativas entre os respondentes da pesquisa e os tipos de processos de inovação aberta adotadas pelas DHs estudadas, já que o padrão das respostas tanto das empresas quanto dos especialistas da área, no que diz respeito aos processos de inovação aberta adotados pelas DHs, não foi significativamente diferente.

Com relação às limitações do estudo destaca-se a impossibilidade de abordar a população em sua totalidade, ou seja, todas as vinte e duas empresas vinculadas ao programa Cl-Brasil, já que algumas dessas empresas haviam paralisado ou encerrado suas atividades, dificultando a possibilidade de contatar as mesmas. Quanto às propostas para estudos futuros, destacam-se realizar um estudo de caso para estudar individualmente, e com maior profundidade, os tipos de inovação aberta adotadas pelas design houses; e comparar os tipos de inovação aberta adotadas pelas DHs vinculadas ao programa $\mathrm{Cl}$-Brasil com aquelas adotadas pelas design houses do setor privado.

\section{REFERÊNCIAS}

ABDI. Agência Brasileira de Desenvolvimento Industrial. As design houses (DHs) brasileiras: relatório analítico. Brasília: ABDI, 2011.

ABDI. Agência Brasileira de Desenvolvimento Industrial. Avaliação das estratégias de negócios das empresas de Projeto de Circuitos Integrados do Programa Cl-Brasil. Brasília: ABDI, 2014.

AITA, B. H.. A cadeia produtiva da indústria de semicondutores: Um estudo exploratório. Dissertação
(Mestrado em Engenharia de Produção) - Universidade Federal do Rio Grande do Sul, Porto Alegre, 2013.

ASAKAWA, K.; SONG, J.; KIM, S.. Open Innovation in Multinational Corporations. In: CHESBROUGH, H.; VANHAVERBEKE, W.; WEST, J.. New frontiers in open innovation. New York: Oxford University Press, 2014. p.157168.

BABBIE, E.. Métodos de pesquisas de Survey. Belo Horizonte: EDUFMG, 2003. 
BAHINIPATI, B. K.; DESHMUKH, S. G.. Vertical collaboration in the semiconductor industry: a decision framework for supply chain relationships. Computers \& Industrial Engineering, v.62, n.2, p.504-526, 2012. DOI:

https://doi.org/10.1016/j.cie.2011.10.017

BRASIL. Ministério da Ciência e Tecnologia. Programa Nacional de Microeletrônica: contribuições para a formulação de um Plano Estruturado de Ações. Brasília: MCT, 2002.

BROWN, C.; LINDEN, G.. Chips and change: how crisis reshapes the semiconductor industry. Cambridge: MIT Press, 2009.

CHESBROUGH, H.. Inovação Aberta: como criar e lucrar com a tecnologia. Porto Alegre: Bookman, 2012.

CHESBROUGH, H.. Open innovation: a new paradigm for understanding industrial innovation. In: CHESBROUGH, H.; VANHAVERBEKE, W.; WEST, J.. Open innovation: researching a new paradigm. New York: Oxford University Press, 2011. p.1-12.

CHESBROUGH, H.; BOGERS, M.. Explicating open innovation: clarifying an emerging paradigm for understanding innovation. In: CHESBROUGH, H.; VANHAVERBEKE, W.; WEST, J.. New frontiers in open innovation. New York: Oxford University Press, 2014. p.3-28.

DAHLANDER, L.; GANN, D. M.. How open is innovation?. Research Policy, v.39, n.6, p.699-709, 2010. DOI: https://doi.org/10.1016/j.respol.2010.01.013

ENKEL, E.; GASSMANN, O.; CHESBROUGH, H.. Open R\&D and open innovation: exploring the phenomenon. R\&D Management, v.39, n.4, p.311-316, 2009. DOI: http://doi.org/0.1111/j.1467-9310.2009.00570.x

FACCIN, K.; BALESTRIN, A.. Práticas Colaborativas em P\&D: Um Estudo na Indústria Brasileira de Semicondutores. Revista de Administração Mackenzie, v.16, n.6, p.190-219, 2015. DOI: http://doi.org/10.1590/167869712015/administracao.v16n6p190-219

FACCIN, K.; BALESTRIN, A.; BORTOLASO, I.. The joint R\&D project: The case of the first Brazilian microcontroller chip. Revista de Administração, São Paulo, v.51, n.1, p.87102, 2016. DOI: http://doi.org/10.5700/rausp1225

FACCIN, K.; BORTOLASO, I.; BALESTRIN, A.. A visão relacional de políticas de ciência e tecnologia: o caso do Programa $\mathrm{Cl}$ Brasil. Revista Eletrônica de Administração, Porto Alegre, v.22, n.1, p.226-251, 2016. DOI:

http://doi.org/10.1590/1413-2311.070142014.54831

FIELD, A. P.. Descobrindo a estatística usando o SPSS. 2 ed. Porto Alegre: Artmed, 2009.

GASSMANN, O.; ENKEL, E.. Towards a theory of open innovation: three core process archetypes. Paris: RADMA, 2004.

HAUSER, G.; ZEN, A. C.; SELAO, D. C.; GARCIA, P. L.. A indústria eletrônica no Brasil e na China: um estudo comparativo e a análise das políticas públicas de estímulo a capacidade tecnológica do setor. Journal of Technology Management \& Innovation, v.2, n.3, p.85-96, 2007.
HILL, M. M.; HILL, A.. Investigação por questionário. 2 ed. Lisboa: Sílabo, 2012.

HUIZINGH, E. K. R. E.. Open innovation: State of the art and future perspectives. Technovation, v.31, n.1, p.2-9, 2011. DOI: https://doi.org/10.1016/i.technovation.2010.10.002

KAPOOR, R.; MCGRATH, P. J.. Unmasking the interplay between technology evolution and R\&D collaboration: Evidence from the global semiconductor manufacturing industry, 1990-2010. Research Policy, v.43, n.3, p.555-569, 2014. DOI: https://doi.org/10.1016/j.respol.2013.08.002

LANGE, K.; MÜLLER-SEITZ, G.; SYDOW, J.; WINDELER, A.. Financing innovations in uncertain networks: Filling in roadmap gaps in the semiconductor industry. Research Policy, v.42, n.3, p.647-661, 2013. DOI: https://doi.org/10.1016/j.respol.2012.12.001

LAURSEN, K.; SALTER, A.. Open for innovation: the role of openness in explaining innovation performance among UK manufacturing firms. Strategic Management Journal, v.27, n.2, p.131-150, 2006. DOI: http://doi.org/10.1002/smj.507

LICHTENTHALER, U.; LICHTENTHALER, E.. A capability-based framework for open innovation: Complementing absorptive capacity. Journal of Management Studies, v.46, n.8, p.13151338, 2009. DOI: http://doi.org10.1111/j.14676486.2009.00854.x

LIMA, R. R. S.; TEIXEIRA, I.; AZEN, C. E.; MIGUEL, H.; SALES, J. R.. Microeletrônica: qual é a ambição do Brasil?. BNDES Setorial, Rio de Janeiro, n.41, p.345- 396, 2015.

MÜLLER-SEITZ, G.; SYDOW, J.. Open innovation at the interorganizational network level: Collaborative Practices in a Semiconductor Industry Consortium. London: 2012.

NIGRO, G.. The effect of early or late R\&D inbound alliance on innovation. Journal of Business Research, v.69, n.5, p.1791-1795, 2016. DOI: https://doi.org/10.1016/j.jbusres.2015.10.057

OLIVEIRA, S. R.; BALESTRIN, A.. Cooperação universidadeempresa: um estudo do projeto UNISINOS-HT Micron para o desenvolvimento de capacidade absortiva na área de semicondutores. Gestão \& Produção, v.25, n.3, p.595-609, 2015. DOI: http://doi.org/10.1590/0104-530X1018-13

PÉNIN, J.; HUSSLER, C.; BURGER-HELMCHEN, T.. New shapes and new stakes: a portrait of open innovation as a promising phenomenon. Journal of Innovation Economics \& Management, v.1, n.7, p.11-29, 2011. DOI: http://doi.org/10.3917/jie.007.0011

PERRI, A.; ANDERSSON, U.. Knowledge outflows from foreign subsidiaries and the tension between knowledge creation and knowledge protection: Evidence from the semiconductor industry. International Business Review, v.23, n.1, p.63-75, 2014. DOI: https://doi.org/10.1016/j.ibusrev.2013.08.007

SAUNDERS, M; LEWIS, P.; THORNHILL, A.. Research Methods for Business Students. 5 ed. London: Pearson Education, 2009. 
SCHNECKENBERG, D.. Open innovation and knowledge networking in a multinational corporation. Journal of Business Strategy, v.36, n.1, p.14-24, 2015. DOI: https://doi.org/10.1108/JBS-08-2013-0066

SEGARRA-CIPRÉS, M.; BOU-LLUSAR, J. C.; ROCA-PUIG, V.. Exploring and exploiting external knowledge: The effect of sector and firm technological intensity. Innovation, v.14, n.2, p.203-217, 2012. DOI:

https://doi.org/10.5172/impp.2012.14.2.203

SIMARD, C.; WEST, J.. Knowledge networks and the geographic locus of innovation. In: CHESBROUGH, H.; VANHAVERBEKE, W.; WEST, J.. Open innovation: researching a new paradigm. New York: Oxford University Press, 2011. p.220-240.

SMIRNOVA, M. M.; PODMETINA, D.; VÄÄTÄNEN, J.; KOUCHTCH, S.. Key stakeholders' interaction as a factor of product innovation: the case of Russia. International Journal of Technology Marketing, v.4, n.3, p.230-247, 2009. DOI: https://doi.org/10.1504/IJTMKT.2009.026872

SONG, M.. A dynamic analysis of cooperative research in the semiconductor industry. International Economic Review, v.52, n.4, p.1157-1177, 2011. DOI:

http://doi.org/10.1111/j.1468-2354.2011.00663.x

TIDD, J.; BARNES, S.. Spin-in or spin-out? Corporate venturing in life sciences. The International Journal of Entrepreneurship and Innovation, v.1, n.2, p.109-116, 2000.
VANHAVERBEKE, W.; DU, J.; LETEN, B.; AALDERS, F.. Exploring Open Innovation at the Level of R\&D Projects. In: CHESBROUGH, H.; VANHAVERBEKE, W.; WEST, J.. New frontiers in open innovation. New York: Oxford University Press, 2014. p.115-131.

VRANDE, V. V.; JONG, J. P. J.; VANHAVERBEKE, W.; ROCHEMONT, M.. Open innovation in SMEs: trends, motives and management challenges. Technovation, v.29, n.6, p.423-437, 2009. DOI: http://doi.org/10.1016/j.technovation.2008.10.001

WANG, C.; CHIU, C.. Competitive strategies for Taiwan's semiconductor industry in a new world economy. Technology in Society, v.36, p.60-73, 2014. DOI: https://doi.org/10.1016/j.techsoc.2013.12.002

WU, C.; DING, C. G.; JANE, T.; LIN, H.; WU, C.. Lessons from the global financial crisis for the semiconductor industry. Technological Forecasting and Social Change, v.99, p.47-53, 2015. DOI:

https://doi.org/10.1016/j.techfore.2015.06.036

ZAHRA, S. A.; NIELSEN, A. P.. Sources of capabilities, integration and technology commercialization. Strategic Management Journal, v.23, n.5, p.377-398, 2002. DOI: http://doi.org/10.1002/smj.229 